\title{
CONJUGACY IN ABELIAN-BY-CYCLIC GROUPS
}

\section{JAMES BOLER}

\begin{abstract}
It is shown that each finitely generated torsion-free abelian-bycyclic group has solvable conjugacy problem. This is done by showing that solving the conjugacy problem for these groups is equivalent to a certain decision problem for modules over the complex group algebra of an infinite cyclic group.
\end{abstract}

1.1 Introduction. Let $G$ be an abelian-by-cyclic group. That is, $G$ has an abelian normal subgroup $A$ with $G / A=T$ cyclic. If $G$ is finitely generated, it can be recursively presented, so it is meaningful to ask whether $G$ has solvable conjugacy problem. Our aim is to answer this question affirmatively when $G$ is torsion-free. More precisely, we prove

THEOREM 1. Let $A$ be an abelian normal subgroup of the finitely generated group $G$. If $G / A=T$ is cyclic and $A$ has no elements of finite order, then $G$ has solvable conjugacy problem.

1.2 Preliminaries. We use the standard notation

$$
x^{y}=y^{-1} x y, \quad[x, y]=x^{-1} y^{-1} x y .
$$

If $R$ is a commutative ring with identity and $T$ is a group, $R T$ is the $R$ algebra which is additively the free $R$-module with basis $T$, with multiplication induced by the multiplication in $T$. In particular, $Z T$ is the integral group ring of $T$ and $\mathbf{C} T$ is the complex group algebra of $T$. If $T$ is infinite cyclic, $\mathbf{C} T$ is a principal ideal domain.

Let $A$ be an abelian normal subgroup of a group $G$ and let $T=G / A$. Conjugation in $G$ induces an action of $T$ on $A$ which gives $A$ the structure of a $Z T$-module. If we write $A$ additively, then $a t=g^{-1}$ ag where $g \in G$ is such that $g A=t$.

If $G$ is finitely generated and $T$ is finitely presented, $A$ is finitely generated as a $Z T$-module.

If $T$ is infinite cyclic, the short exact sequence $0 \rightarrow A \rightarrow G \rightarrow T \rightarrow 1$ splits, so that $G$ is isomorphic with the semidirect product $A] T$ of the $Z T$-module $A$ by $T$. Thus $T$ may be regarded as a subgroup of $G$.

If $A$ is a $Z T$-module, $A \otimes_{Z} \mathbf{C}$ becomes a $\mathbf{C} T$-module via $(a \otimes 1) t=a t \otimes 1$. If $A$ is generated as a $Z T$-module by $a_{1}, \ldots, a_{k}, A \otimes \mathbf{C}$ is generated as a $\mathbf{C} T$ module by $a_{1} \otimes 1, \ldots, a_{k} \otimes 1$. If $A$ is torsion-free, the map $a \mapsto a \otimes 1$ defines an embedding (of abelian groups) of $A$ into $A \otimes \mathbf{C}$.

Received by the editors February 25, 1975.

AMS (MOS) subject classifications (1970). Primary 20F10; Secondary 16A26.

Key words and phrases. Conjugacy problem, group algebra, group ring. 
1.3 A module-theoretic decision problem. Let $R$ be a commutative ring with identity, $T$ a group and $A$ a finitely presented $R T$-module. We say $A$ has solvable conjugacy problem if there is an effective procedure for determining whether, for $a$ and $b$ in $A$, there is $t \in T$ with $a t=b$.

THEOREM 2. Let $T$ be cyclic and $A$ a finitely generated ZT-module without elements of finite order. Then $A$ has solvable conjugacy problem.

To prove Theorem 2, notice first that if $T$ is a finite cyclic group, $A$ is finitely generated as an abelian group and so $A] T$ is polycyclic. By [2] $A] T$ has solvable conjugacy problem, so $A$ has solvable conjugacy problem. Thus we may assume $T$ is infinite cyclic.

Now, since $A$ is torsion-free, the map $a \mapsto a \otimes 1$ induces an embedding of $A$ into $A \otimes \mathbf{C}$, and it is enough to show that the $\mathbf{C} T$-module $A \otimes \mathbf{C}$ has solvable conjugacy problem.

Since $\mathbf{C} T$ is a principal ideal domain, there is a decomposition

$$
A \otimes \mathbf{C}=A_{1} \oplus \cdots \oplus A_{k}
$$

where each $A_{i}(1 \leqq i \leqq k)$ is a nonzero cyclic submodule of $A \otimes \mathbf{C}$. Clearly, then, it is enough to show that a cyclic $\mathbf{C} T$-module has solvable conjugacy problem.

Let $A_{i} \approx \mathrm{C} T / J_{i}(1 \leqq i \leqq k)$. We distinguish between the cases $J_{i}$ $=(0)$ and $J_{i} \neq(0)$.

1.4 Free submodules. If $J_{i}=(0), A_{i} \approx \mathrm{C} T$ is a free submodule of rank 1 .

To see that $\mathbf{C T}$ has solvable conjugacy problem, let $t$ be a generator for $T$. Then there are unique representations

$$
a=c_{1} t^{s_{1}}+\cdots+c_{p} t^{s_{p}}, \quad b=d_{1} t^{w_{1}}+\cdots+d_{q} t^{w_{q}}
$$

with

$$
\begin{aligned}
& 0 \neq c_{i} \in \mathbf{C} \quad(1 \leqq i \leqq p) \quad \text { and } \quad s_{1}<\cdots<s_{p}, \\
& 0 \neq d_{i} \in \mathbf{C} \quad(1 \leqq i \leqq q), \quad w_{1}<\cdots<w_{q} .
\end{aligned}
$$

To decide whether $a t^{l}=b$ for some $l \in Z$, simply notice that the only possibility for $l$ is $w_{q}-s_{p}$. It is clearly possible, for a fixed power $t$ of $t$, to effectively decide whether $a t^{l}=b$.

1.5 Finite-dimensional submodules. If $J_{i} \neq(0), \operatorname{dim}_{\mathbf{C}} A_{i}=n<\infty$. The action of a generator $t$ of $T$ on a basis of the vector space $A_{i}$ yields an invertible $n \times n$ matrix $M$ in the usual way. Notice that the matrix $M$ is effectively computable from a presentation of $A_{i}$.

Recall that if $G$ is a finitely presented group and the word problem is solvable for some finite presentation $P_{1}$ of $G$, then it is also solvable for any other finite presentation $P_{2}$ of $G$ (cf. [4]). A similar argument shows that if the conjugacy problem is solvable for some finite presentation $P_{1}$ of a $\mathrm{C} T$-module $A$, it is solvable for any other presentation $P_{2}$ of $A$. Because of this we may assume that the module $A_{i}$ is presented so that the matrix $M$ is in Jordan canonical form (cf. [3]). Thus 


$$
M=\left(\begin{array}{cccc}
J_{1} & & & \\
& J_{2} & & \\
& & \ddots & \\
& & & J_{q}
\end{array}\right)
$$

where each $J_{i}(1 \leqq i \leqq q)$ is of the form

$$
J_{i}=\left(\begin{array}{ccccc}
\lambda & 1 & . & \cdots & 0 \\
0 & \lambda & 1 & \cdots & 0 \\
\cdot & \cdot & \cdot & \cdots & \cdot \\
\cdot & \cdot & \cdot & \cdots & \cdot \\
. & . & . & \cdots & . \\
\cdot & . & . & \cdots & 1 \\
0 & . & . & \cdots & \lambda
\end{array}\right) .
$$

Let

$$
a=\left(\begin{array}{c}
a_{1} \\
\vdots \\
a_{n}
\end{array}\right) \text { and } b=\left(\begin{array}{c}
b_{1} \\
\vdots \\
b_{n},
\end{array}\right), \quad a_{i}, b_{i} \in C \quad(1 \leqslant i \leqslant n) \text {. }
$$

Our aim is to show there is an effective process for determining whether $M^{r} a=b$ for some positive integer $r$. Since

$$
M=\left(\begin{array}{llll}
J_{1}^{r} & & & \\
& J_{2}^{r} & & \\
& & \ddots & \\
& & & J_{q}^{r}
\end{array}\right)
$$

it is not hard to see that we may assume $k=1$ so that $M$ can be taken to be the $n \times n$ matrix

$$
M=\left(\begin{array}{ccccc}
\lambda & 1 & 0 & \cdots & 0 \\
0 & \lambda & 1 & \cdots & . \\
\cdot & \cdot & . & \cdots & . \\
\cdot & . & . & \cdots & . \\
. & . & . & \cdots & . \\
. & . & . & \cdots & 1 \\
0 & . & . & \cdots & \lambda
\end{array}\right)=\lambda I+S
$$

where

$$
S=\left(\begin{array}{ccccc}
0 & 1 & 0 & \cdots & 0 \\
0 & 0 & 1 & \ldots & 0 \\
. & . & . & \ldots & . \\
. & . & . & \cdots & . \\
. & . & . & \ldots & . \\
. & . & . & \cdots & 1 \\
0 & . & . & \cdots & 0
\end{array}\right)
$$


Here, $\lambda \neq 0$ since $M$ is invertible.

$$
M^{r}=(\lambda I+S)^{r}=\sum_{k=0}^{r}\left(\begin{array}{l}
r \\
k
\end{array}\right) \lambda^{r-k} S^{k}
$$

Since $S^{n}=0$, we have for $r \geqq n$,

$$
n^{r}=\sum_{k=0}^{n-1}\left(\begin{array}{l}
r \\
k
\end{array}\right) \lambda^{r-k} S^{k} .
$$

To effectively decide whether $M^{r} a=b$ for some $r$, then, we must be able to decide in a finite number of steps whether there is a solution to the system of equations:

$$
\begin{aligned}
& \lambda^{r} a_{1}+r \lambda^{r-1} a_{2}+\cdots \\
& \begin{array}{c}
\lambda^{r} a_{2}+\cdots \\
\vdots
\end{array}
\end{aligned}
$$

(n)

$$
\begin{aligned}
\cdots+\left(\begin{array}{c}
r \\
n-1
\end{array}\right) \lambda^{r-n+1} a_{n} & =b_{1}, \\
\cdots+\left(\begin{array}{c}
r \\
n-2
\end{array}\right) \lambda^{r-n+2} a_{n} & =b_{2}, \\
\vdots & \\
\lambda^{r} a_{n-1}+\quad r \lambda^{r-1} a_{n} & =b_{n-1,} \\
\lambda^{r} a_{n} & =b_{n} .
\end{aligned}
$$

We can obtain a solution as follows. Suppose first that $|\lambda| \neq 1$, and consider equation $(n): \lambda^{r} a_{n}=b_{n}$.

As $r$ increases without bound, $\left|\lambda^{r}\right|$ either increases without bound or approaches 0 . Either way, if $a_{n} \neq 0$ there are only finitely many values of $r$ which we need check and we can check each of these values simply by computing $M^{r} a$. If $a_{n}=0$, consider equation $(n-1)$, which becomes $\lambda^{r} a_{n-1}$ $=b_{n-1}$.

Again, considering the cases $a_{n-1} \neq 0$ and $a_{n-1}=0$, we can inductively obtain a decision in a finite number of steps.

Now assume $|\lambda|=1$. By an induction argument, we may assume $a_{n} \neq 0$. Consider equation $(n-1): \lambda^{r} a_{n-1}+r \lambda^{r-1} a_{n}=b_{n-1}$. Since $a_{n} \neq 0$ and $|\lambda|$ $=1,\left|\lambda^{r} a_{n-1}+r \lambda^{r-1} a_{n}\right|$ increases without bound as $r$ increases without bound. Thus, there are again only finitely many values of $r$ for which we need compute $M^{r} a$.

We have shown that we can effectively decide whether $M^{r} a=b$ for $r \geqq 1$. By considering $\left(M^{-1}\right)^{r}=M^{-r}$; we can decide in a finite number of steps whether $M^{r} a=b$ for any $r \in Z$. This completes the proof of Theorem 2 .

1.6 Proof of Theorem 1. Let $G, A$ and $T$ be as in the statement of Theorem 1. We have seen that we may assume $T$ is infinite cyclic, so that $G=A] T$. Let $g_{1}, g_{2} \in G$. If $g_{1} \neq g_{2} \bmod A$, then $g_{1}$ and $g_{2}$ are not conjugate in $G$. Thus there are unique representations

$$
g_{1}=a s \quad \text { and } \quad g_{2}=b s \quad(a, b \in A, s \in T) .
$$

To decide whether $g_{1}$ is conjugate to $g_{2}$ by an element $g_{3}=c t(c \in A, t$ $\in T$ ), we must check whether $g_{1}^{g_{3}}=(a s)^{c t}=b$. Now

$$
(a s)^{c t}=a^{t}\left(c^{-1} s c\right)^{t}=\left(a^{t} c^{-t} c^{s^{-1} t}\right) s .
$$


If we write $A$ additively, we have $g_{1}$ conjugate to $g_{2}$ if and only if there is a solution $c \in C, t \in T$ to the equation $a t+\left(s^{-1}-1\right) t=b$.

When we look at this equation modulo the normal subgroup $N$ of $G$ generated by $s$ (notice that $N \cap A$ is the submodule of $A$ generated by $\left.\left(s^{-1}-1\right)\right)$, we see that there is a solution if and only if $a t=b \bmod N$.

If $N \neq 1$, then $G / N$ is polycyclic and so has solvable conjugacy problem. If $N=1$, then $s=1$ and invoking Theorem 2 proves that $G$ has solvable conjugacy problem. This completes the proof of Theorem 1 .

ACKNOWLEDGEMENTS. The results of this paper grew out of the author's thesis [1]. I should like to take this opportunity to thank my supervisor Gilbert Baumslag for his help.

\section{REFERENCES}

1. J. Boler, Embedding and conjugacy in metabelian groups, Thesis, Rice University, 1974.

2. E. Formanek, Matrix techniques in polycyclic groups, Thesis, Rice University, 1970.

3. I. N. Herstein, Topics in algebra, Blaisdell, New York and Toronto, 1964, p. 258. MR 30 $\# 2028$.

4. W. Magnus, A. Karass and D. Solitar, Combinatorial group theory: Presentations of groups in terms of generators and relations, Pure and Appl. Math., vol. 8, Interscience, New York, 1966, p. 55. MR 34 \#7617.

Department of Mathematics, Oklahoma State University, Stillwater, Oklahoma 74074 